\title{
The use of cladribine in the treatment of multiple sclerosis
}

\author{
Authors: Nikola Erceg ${ }^{1}$, Anamaria Dukić ${ }^{1}$, Tereza Gabelić ${ }^{1,2}$ (mentor) \\ ${ }^{1}$ School of Medicine, University of Zagreb, Croatia \\ ${ }^{2}$ University Hospital Centre Zagreb, Department of Neurology, Croatia \\ DOI: https://doi.org/10.26800/LV-142-supp5-14
}

\section{Background:}

Multiple sclerosis (MS) is a chronic inflammatory disease that affects the central nervous system. The cause of MS is still unknown, but it is considered to be multifactorial. A combination of clinical examination, radiological, and laboratory methods according to the McDonald diagnostic criteria confirms the diagnosis with high probability. Management of MS includes disease-modifying drugs that show different levels of efficacy. Cladribine is an antimetabolite drug indicated for the treatment of highly active relapsing-remitting MS based on clinical or imaging features.

\section{Case presentation:}

A 45-year-old female patient was diagnosed with MS in 2014. She presented with right-sided optic neuritis. Magnetic resonance imaging (MRI) of the brain and orbits revealed multiple supra and infratentorial demyelinating lesions and hyperintensity of the right optic nerve. Visual evoked potentials demonstrated conduction block on the right side. Cerebrospinal fluid analysis displayed intrathecal synthesis. Treatment with dimethyl fumarate was introduced. On the control MRI in June 2018, there was a newly formed lesion with post-contrast imbibition at the C3-C4 segment of the spinal cord. Due to the exacerbation of the disease, cladribine therapy was introduced in 2018 with a good clinical response. MRI from August 2019 showed no new lesions. The patient received the second cycle of cladribine in August and September 2019. The last control MRI from May 2020 showed one new juxtacortical non-active lesion at the tip of the right temporal lobe without clinical correlation.

\section{Conclusion:}

As a purine analogue with chloride substituent, cladribine targets lymphocytes and leads to their apoptosis. The criteria for initiating therapy is lymphocyte count in the reference interval. Contraindications are HIV infection, active chronic infection, active malignant disease, and creatinine clearance $<60 \mathrm{~mL} / \mathrm{min}$. Laboratory, imaging and clinical follow up are mandatory in monitoring therapeutic response and possible side-effects of the treatment.

Keywords: Cladribine, Lymphocyte count, Multiple sclerosis 